\title{
Insulin resistance in the vasculature
}

\author{
Kieren J. Mather, ${ }^{1}$ Helmut O. Steinberg, ${ }^{2}$ and Alain D. Baron ${ }^{3}$ \\ ${ }^{1}$ Indiana University School of Medicine, Indianapolis, Indiana, USA. ${ }^{2}$ Merck Research Laboratories, Rahway, New Jersey, USA. \\ ${ }^{3}$ Elcelyx Therapeutics Inc., San Diego, California, USA.
}

\begin{abstract}
Insulin resistance is typically defined as a reduced ability of insulin to induce glucose uptake by target tissues such as fat and skeletal muscle cells. It accompanies several disease states, including obesity, type 2 diabetes, hepatitis $\mathrm{C}$, and polycystic ovary syndrome, and is a primary feature of metabolic syndrome. Outside of its effects on blood glucose levels, insulin resistance is also associated with a 2- to 3-fold increased risk of cardiovascular mortality. In 1996, Alain Baron, Helmut Steinberg, and colleagues demonstrated that insulin resistance is associated with endothelial dysfunction. This seminal observation led to significant advances in our understanding of insulin's action in health and disease.
\end{abstract}

In the mid-1990s, our lab produced seminal observations demonstrating that insulin-mediated augmentation of skeletal muscle blood flow was a physiologic feature of insulin's action in humans (1). Moreover, in the insulin-resistant states of human obesity and type 2 diabetes, this vascular action of insulin was impaired (2). These studies used femoral arterial catheterizations to evaluate the response to intra-arterial vasodilator stimuli, comparing control conditions against euglycemic-hyperinsulinemic clamp conditions. Obesity was associated with parallel defects in the vasodilatory responses to insulin and to methacholine, an endothelium-dependent vasodilator. We further demonstrated that the vasodilatory effects of insulin were mediated by the release of endothelial nitric oxide, and we and others demonstrated that blocking nitric oxide production in vivo could induce insulin resistance and inhibit glucose uptake by preventing insulin-mediated vasodilation in skeletal muscle (3). Finally, we demonstrated that endothelial dysfunction, characterized by reduced nitric oxide production and exaggerated release of endothelin, was a key feature of human insulin-resistant states (4). The link between insulin resistance and endothelial dysfunction is now widely accepted, and there is broad recognition that insulin's action to enhance its own vascular delivery (and that of its substrates) is integral to its overall action.

Conflict of interest: The authors have declared that no conflict of interest exists.

Citation for this article: J Clin Invest. 2013; 123(3):1003-1004. doi:10.1172/JCI67166
These observations have prompted much subsequent work, including the exploration of the cellular and molecular details of the vascular actions of insulin. Quon and colleagues demonstrated that the molecular signaling pathways mediating insulin stimulation of nitric oxide production in endothelial cells are similar to signaling pathways mediating insulin activation of glucose transport in classical target tissues (i.e., the PI 3-kinase pathway) (5). Among many experiments confirming these observations, mice with defective insulin signaling (Irs1 knockout) exhibited impaired endothelial function and insulin resistance (6). While insulin in endothelial cells also acts via a MAP kinase pathway independent of impaired insulin receptor/IRS-1 signaling (5), in the setting of impaired endothelial IRS-1 function, insulin preferentially activates the MAP kinase pathway, resulting in a mitogenic, proatherosclerotic response in the vascular wall. These effects are prominently driven by endothelin. Excess endothelin action appears to contribute to insulin resistance in human obesity (4). A current model suggests that endothelial insulin sensitivity determines a balance between the vasculoprotective PI 3-kinase and proatherogenic MAP kinase pathways (Figure 1).

The observation that insulin's vasodilatory actions are impaired in obesity and insulin resistance has also prompted studies exploring the location of this action in the vascular tree. Using a clever microbubble technique, the Barrett and Clark laboratories demonstrated that insulin acts in a nitric oxide-dependent fashion to regulate capillary recruitment and perfusion by affecting precapillary resistance vessels
(7). Importantly, this action of insulin precedes the induction of glucose uptake (8), demonstrating that the vascular effects of insulin are primary and do not arise as a consequence of changes in cellular metabolism. These same authors have confirmed that insulin resistance is associated with impaired capillary recruitment and altered skeletal muscle perfusion.

Recognition of impaired vascular actions of insulin in obesity and insulin resistance has recast prior observations of the contributions of fatty acids and inflammation to systemic insulin resistance and the role of other metabolically active hormones on the vasculature. Fatty acid excess (particularly palmitic acid), previously known to cause insulin resistance, also induces tissue and vascular insulin resistance (9). Inflammation is associated with vascular dysfunction, and fatty acids appear to act in part by inducing local inflammation. Fatty acid lowering achieved with nicotinic acid or with PPAR $\gamma$ agonists has proven beneficial for vascular function and for insulin sensitivity, but the presence of multiple concurrent effects prevents simple assessment of underlying causal relationships. Therapeutically reducing systemic inflammation has been surprisingly ineffective at improving vascular function in diabetes. In general, interventions that improve insulin action have also been shown to ameliorate endothelial function. Whether improved endothelial function could result in improved insulin action has not been shown.

The effects of other fuel hormones on the vasculature have also been evaluated. Most recently, studies with glucagon-like peptide 1 (GLP-1) have shown that it can act similarly to insulin as a microvascular vasodilator in skeletal muscle (10). Additionally, GLP-1 appears to regulate myocardial perfusion and fuel selection (observations that are leading to clinical studies of GLP- 1 in the setting of myocardial ischemia). Further study is required to evaluate the impact of diabetes and obesity on the vascular effects of GLP-1 and other fuel hormones, and to evaluate the therapeutic application of these observations. 


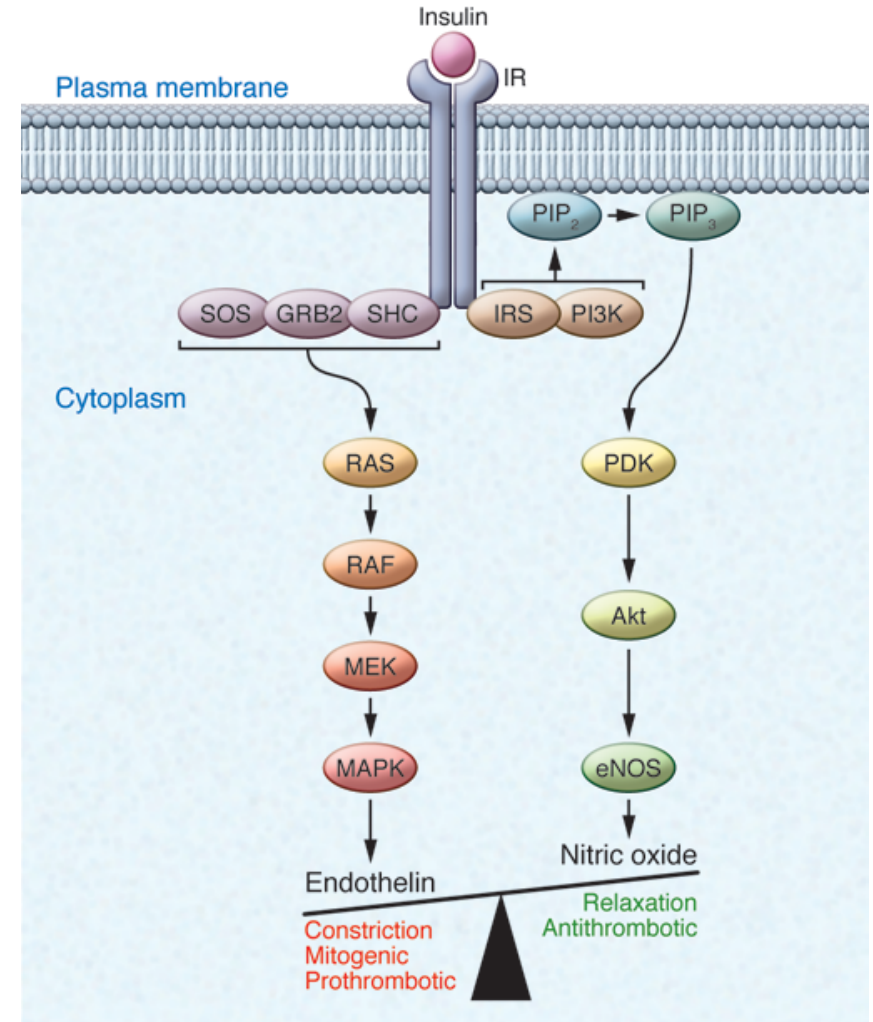

Much remains to be learned regarding insulin's vascular effects and the role of endothelial insulin resistance in the pathogenesis of atherosclerosis. It is interesting to speculate that correction of endothelial vascular resistance, independent of insulin action in other tissues, may be sufficient to reduce the risk of insulin resistance-associated vascular disease.

Address correspondence to: Alain Baron, Elcelyx Therapeutics Inc., 11995 El Camino Real, Ste. 305, San Diego, California 92130, USA. Phone: 858.344.2816; Fax: 858.436.2126; E-mail: alain.baron@elcelyx.com.
1. Baron AD, Steinberg HO, Chaker H, Leaming R, Johnson A, Brechtel G. Insulin-mediated skeletal muscle vasodilation contributes to both insulin sensitivity and responsiveness in lean humans. J Clin Invest. 1995;96(2):786-792.

2. Steinberg HO, Chaker H, Leaming R, Johnson A, Brechtel G, Baron AD. Obesity/insulin resistance is associated with endothelial dysfunction. Implications for the syndrome of insulin resistance. J Clin Invest. 1996;97(11):2601-2610.

3. Steinberg HO, Brechtel G, Johnson A, Fineberg N, Baron AD. Insulin-mediated skeletal muscle vasodilation is nitric oxide dependent. A novel action of insulin to increase nitric oxide release. J Clin Invest. 1994;94(3):1172-1179.

4. Lteif A, Vaishnava P, Baron AD, Mather KJ. Endothelin limits insulin action in obese/insulin-resistant humans. Diabetes. 2007;56(3):728-734.

5. Muniyappa R, Montagnani M, Koh KK, Quon MJ. Cardiovascular actions of insulin. Endocr Rev.

\section{Figure 1}

Distinct signaling pathways mediate insulin effects on nitric oxide and endothelin in vascular endothelial cells. Endothelial insulin resistance can preferentially impair signaling via the IRS/PI3K pathway, adversely affecting the balance between prothrombotic and antithrombotic signaling. This imbalance could potentially contribute to the increased cardiovascular mortality associated with insulin resistance.
2007;28(5):463-491.

6. Abe $\mathrm{H}$, et al. Hypertension, hypertriglyceridemia, and impaired endothelium-dependent vascular relaxation in mice lacking insulin receptor substrate-1. J Clin Invest. 1998;101(8):1784-1788.

7. Clark MG, et al. Blood flow and muscle metabolism: a focus on insulin action. Am J Physiol Endocrinol Metab. 2003;284(2):E241-E258.

8. Vincent MA, et al. Microvascular recruitment is an early insulin effect that regulates skeletal muscle glucose uptake in vivo. Diabetes. 2004; 53(6):1418-1423.

9. Steinberg HO, Paradisi G, Hook G, Crowder K, Cronin J, Baron AD. Free fatty acid elevation impairs insulin-mediated vasodilation and nitric oxide production. Diabetes. 2000;49(7):1231-1238.

10. Chai W, et al. Glucagon-like peptide 1 recruits microvasculature and increases glucose use in muscle via a nitric oxide-dependent mechanism. Diabetes. 2012;61(4):888-896. 